\title{
Etude dynamique des apports en éléments majeurs et nutritifs des eaux de la lagune de Porto-Novo (Sud Bénin)
}

\author{
Daouda MAMA ${ }^{1,2}$, Waris CHOUTI ${ }^{1,2^{*}}$, Abdoukarim ALASSANE ${ }^{2}$, \\ Odilon CHANGOTADE ${ }^{2}$, François ALAPINI ${ }^{1}$ et Moussa BOUKARI ${ }^{2}$ \\ ${ }^{1}$ Laboratoire de Chimie Inorganique et de l'Environnement, Faculté des Sciences et Techniques (FAST), \\ Université d'Abomey-Calavi BP : 4521 Cotonou, Bénin. \\ ${ }^{2}$ Laboratoire d'Hydrologie Appliquée Faculté des Sciences et Techniques (FAST), Université d'Abomey-Calavi \\ 01 BP : 526 Cotonou, Bénin. \\ ${ }^{3}$ Laboratoire d'Expertise et de Recherche en Chimie de l'Eau et de l'Environnement (LERCEE) \\ Faculté des Sciences et Techniques (FAST), Université d'Abomey-Calavi01 BP : 526 Cotonou, Bénin. \\ *Auteur correspondant ; E-mail : lawaniwaris@yahoo.fr; Tél : 0022997487320
}

\section{RESUME}

Afin d'évaluer le niveau trophique de la lagune qui expliquerait la prolifération des algues sur la lagune, les nutriments (NTK et phosphore total) et la Demande Chimique en Oxygène (DCO) des eaux de la lagune Porto-Novo ont été déterminés suivant les quatre saisons de l'année. L'étude a également permis d'analyser par chromatographie les éléments minéraux, anions et cations, dans les eaux de la lagune. Les résultats ont montré que les concentrations en chlore (variant entre 0,84 et $1180 \mathrm{mg} / \mathrm{L}$ ) et en sodium (variant entre 35,52 et 7305 $\mathrm{mg} / \mathrm{L}$ ) sont plus élevées au cours de la période de basses eaux; les eaux marines arrivent dans la lagune par l'intermédiaire du lac Nokoué. Les concentrations obtenues au cours de la période des basses eaux pour les NTK montrent que le Canal de Totchè et la savonnerie de l'Industrie Béninoise des Corps Gras (IBCG) sont très riches en NTK. De même, les concentrations élevées pour le phosphore total et les phosphates sont obtenues au début de la saison des pluies, la concentration la plus élevée est obtenue à l'IBCG. Les valeurs moyennes annuelles de phosphore total dans les eaux varient de $0,31 \mathrm{mg} / \mathrm{L}$ à $6,05 \mathrm{mg} / \mathrm{L}$, elles dépassent donc $100 \mu \mathrm{gP} / \mathrm{L}$ : la lagune est donc hypereutrophe selon l'Organisation de Coopération et Développement Economique (OCDE, 1982). Quant aux valeurs moyennes de la DCO, elles varient de $101 \mathrm{mgO}_{2} / \mathrm{L}$ à 142 $\mathrm{mgO}_{2} / \mathrm{L}$. Les mesures effectuées au cours des différentes périodes de l'année montrent que les eaux de ruissellement contribuent beaucoup à l'eutrophisation de la lagune. Ceci montre aussi l'urgence de la mise en place d'un système de gestion et de contrôle des eaux usées avant leur déversement dans la lagune.

(C) 2011 International Formulae Group. All rights reserved.

Mots-clés : pollution, phosphore, azote, eaux de ruissellement, eutrophisation

\section{INTRODUCTION}

Les déchets agricoles et ménagers continuent d'être rejetés sans traitement dans les rivières et lacs de plusieurs pays africains, empêchant ainsi ces cours d'eau de jouer pleinement leurs rôles. L'apport des nutriments, notamment du phosphore, dans un milieu aquatique entraîne une augmentation de la productivité de la biomasse des végétaux et des algues qui a des effets indésirables tels 
que: l'accroissement de la turbidité, l'augmentation des apports de matière organique qui mène à une sédimentation élevée, la privation d'oxygène.

Lorsque la croissance excessive de végétaux s'étend à certaines cyanobactéries, il peut $\mathrm{y}$ avoir production de toxines, qui exposent la vie aquatique, le bétail et la santé humaine à un risque accru (Chambers et al., 2001).

Les lagunes sont des écosystèmes très riches en biodiversité. Elles servent d'habitats irremplaçables, de zones de ponte et de nurseries pour de nombreuses espèces (Ruiz et al., 2006). De même, l'importance de ces cours d'eau dans l'économie humaine ne cesse de croître (Mohamedou et al., 2008). Mais ces écosystèmes sont naturellement vulnérables et leur équilibre écologique peut être rapidement modifié sous l'influence de facteurs naturels ou anthropiques (Kouassi, 2005). L'étude de la qualité physicochimique et l'évaluation du niveau trophique des cours d'eau permet de caractériser ces éléments minéraux qui dégradent la qualité des cours d'eau et menacent la vie aquatique.

La lagune de Porto-Novo (sud-Bénin) reçoit régulièrement les déchets liquides domestiques des populations par l'intermédiaire des collecteurs d'eau. Elle constitue une source importante de produits halieutiques. En effet, du point de vue de la production halieutique annuelle, la lagune de Porto-Novo représente le troisième plan d'eau productif du sud-Bénin après le lac Nokoué et le lac Ahémé. Elle est une voie navigable très pratiquée par les populations du village lacustre des Aguégués et autres. Des études antérieures (Chouti, 2010a) ont montré que les teneurs moyennes annuelles en oxygène dissous des eaux de la lagune varient entre 0,89 et $5,41 \mathrm{mg} / \mathrm{L}$ et sont donc inférieures à la concentration minimale acceptable pour les premiers stades biologiques (CCMRE, 1987; Truelson, 1997).

Le diagnostic de l'eutrophisation, qu'effectue le Réseau de Suivi Lagunaire
(RSL) en France, prend en compte cinq compartiments que sont la colonne d'eau, les sédiments, le phytoplancton, la macroflore et la macrofaune, susceptibles d'être des indicateurs du niveau de l'eutrophisation du milieu (Trinquier, 2009).

Dans cette étude, il a été considéré deux compartiments : la colonne d'eau et les sédiments. En effet, la colonne d'eau indique le niveau eutrophe ou anoxique du milieu à travers la mesure des paramètres tels que l'azote, le phosphore, la température et l'oxygène de l'eau. De même, ce compartiment fait apparaître les conséquences de l'eutrophisation, comme la turbidité et la toxicité de l'eau. Les sédiments, par contre, constituent des pièges pour le phosphore et l'azote et lorsque les conditions deviennent favorables ces nutriments sont relargués dans la colonne d'eau. Les sédiments peuvent devenir donc des sources de pollution endogène.

Cette étude vise à connaître les teneurs en éléments minéraux de la lagune et leurs sources. De même, elle cherche à mieux cerner le problème d'eutrophisation de la lagune ainsi qu'à expliquer les teneurs minimales d'oxygène dissous constatées.

\section{MATERIEL ET METHODES \\ Prélèvement des échantillons}

Les points de prélèvements (Figure 1) ont été choisis en tenant compte des déversements domestiques, eaux de ruissellement et apport du Lac Nokoué ; ils sont au nombre de six.

L'échantillonnage a été réalisé comme suit :

- Les flacons en plastique utilisés pour les prélèvements d'eau sont préalablement lavés et rincés au laboratoire, rincés avec l'eau à prélever sur le terrain,

- Le prélèvement d'eau est effectué à environ $5 \mathrm{~cm}$ de la surface,

- Les flacons remplis complètement sont fermés hermétiquement pour éviter toute fuite et entrée de gaz. 


\section{Produits, matériel et méthodes}

Les éléments majeurs (anions et cations) ont été déterminés par un chromatographe ionique DIONEX ICS-1000. Le dosage par chromatographie ionique DIONEX ICS-1000 est une technique analytique qui permet l'analyse qualitative (par séparation des espèces présentes) et quantitative des espèces ioniques présentes dans un échantillon liquide dépourvu de Matières En Suspension. Le Tableau 2 présente les conditions chromatographiques : colonne, éluant, mode d'élution.

Les composés azotés organiques et inorganiques sont transformés en nitrates selon la méthode de Koreleff à l'aide d'un oxydant dans un thermoréacteur. Dans une solution acidifiée avec l'acide sulfurique et phosphorique, les nitrates forment avec le diméthyl-2,6-phénol (DMP) le nitro-4diméthyl-2,6-phénol qui est dosé par photométrie. La minéralisation est analogue à EN ISO 11905-1.

Pour déterminer le phosphore total, à $25 \mathrm{ml}$ d'échantillon, on ajoute $2 \mathrm{ml}$ d'acide sulfurique $(5,25 \mathrm{~N})$ et $\mathrm{du}$ persulfate de potassium. Afin de permettre la minéralisation, l'échantillon est porté à ébullition pendant 30 minutes. Après refroidissement à la température ambiante, on ajoute $2 \mathrm{ml}$ de soude $(5 \mathrm{~N})$ pour neutraliser la préparation et $1 \mathrm{ml}$ de molybdovanadate qui permet la mise en évidence du phosphore : plus la solution est jaune, plus il y a de phosphore. La préparation ainsi obtenue est ensuite placée dans le spectrophotomètre qui mesure le phosphore total. Le résultat est affiché en $\mathrm{mg} / \mathrm{l}$.

Pour déterminer la DCO, à 2,5 ml d'échantillon, on ajoute $1,5 \mathrm{ml}$ de dichromate de potassium $0,04 \mathrm{M}$ et $3,5 \mathrm{ml}$ d'acide sulfurique concentré. Un « blanc » est également réalisé avec $2,5 \mathrm{ml}$ d'eau distillée à la place de l'échantillon. La réaction est pratiquée à chaud $\left(150{ }^{\circ} \mathrm{C}\right)$, durant deux heures. Après refroidissement, la quantité d'oxydant restante est dosée par photométrie.
Cette méthode permet d'obtenir directement la DCO de chaque échantillon en milligrammes d'oxygène $\left(\mathrm{O}_{2}\right)$ par litre.

L'analyse des données est effectuée à l'aide du logiciel SPSS 12.

\section{RESULTATS}

Les résultats obtenus sont présentés dans la suite et concernent les teneurs en anions et cations de la lagune. De même, les teneurs en nutriments et matières organiques ont permis de justifier la prolifération des algues qu'on observe au niveau de la lagune et de comprendre le déficit en oxygène qui en découle.

\section{Les éléments minéraux majeurs Les anions}

Les teneurs des différents anions sont présentées dans la Figure 2. Elle montre que les teneurs de l'ion chlorure sont très élevées alors que celles de l'ion bromure sont très faibles.

L'ion fluorure : la concentration de la lagune en ion fluorure est très faible. Elle est nulle pendant la période de basses eaux et au début des pluies.

L'ion bromure: $\mathrm{Sa}$ concentration est également très faible dans la lagune et n'est présente que pendant la saison sèche.

Les nitrates et nitrites : Les concentrations en nitrate sont faibles par rapport aux normes fixées par les directives européennes (Circulaire DCE $n^{\circ}$ 2005-12 du 28/07/05) et les recommandations canadiennes (Conseil canadien des ministres de l'environnement, 2003).

Certaines mesures de nitrite par contre dépassent largement les directives européennes et on remarque que le taux de nitrite est élevé à Douane Topka. La teneur moyenne annuelle du nitrate $(1,10 \mathrm{mg} / \mathrm{L})$ est inférieure à celle enregistrée dans la lagune de Fresco qui est de 21,31 mg/L (Yacoub et al., 2008). Par contre la teneur moyenne annuelle du nitrite $(0,37 \mathrm{mg} / \mathrm{L})$ est supérieure à celle observée dans la même lagune. 
Les chlorures: Les teneurs moyennes annuelles sont très supérieures à celles observées au lac Dayet Aoua au Maroc (Abba et al., 2008). Les concentrations en chlore sont plus élevées au cours de la période de basses eaux et c'est au Canal de Totchè que les concentrations en chlore sont plus élevées. C'est par ce point que pénètrent les eaux marines dans la lagune.

Les sulfates : Les sulfates sont anormalement élevés à Djassin et à Douane Topka pendant la période des basses eaux.

Les orthophosphates: Les concentrations élevées sont obtenues au début de la saison des pluies. Ces orthophosphates sont donc drainés vers la lagune par les eaux de ruissellement qui constituent de ce fait une source de pollution de la lagune. La teneur moyenne en orthophosphates des eaux de la lagune est supérieure aux teneurs $39 \mu \mathrm{g} / \mathrm{L}$ et $50 \mu \mathrm{g} / \mathrm{L}$ enregistrées respectivement dans le lac Léman (Rapin et al., 1999) et dans le lac Ichkeul (M'Barek, 1995).

Les cations: Les teneurs des différents cations sont présentées dans la Figure 3. Elle montre que les teneurs de l'ion sodium sont très élevées alors que celles du lithium sont très faibles.

Le lithium : La présence du lithium dans la lagune n'est significative qu'en période des hautes eaux à Beaurivage.

Le sodium: Les résultats sont identiques à ceux du chlore à savoir : les concentrations sont plus élevées au cours de la période de basses eaux et c'est le Canal de Totchè qui présente les plus fortes concentrations.

L'ion ammonium : Les concentrations élevées sont observées au cours de la saison sèche. Elles sont supérieures à la limite supérieure du bon état chimique des directives européennes. La teneur moyenne annuelle de $0,72 \mathrm{mg} / \mathrm{L}$ est supérieure à celle enregistrée dans la lagune de Fresco qui est de 0,06 mg/L (Yacoub et al., 2008).

Le potassium : Les concentrations élevées sont obtenues au cours de la période des basses eaux ; ceci est dû à l'apport des eaux salées de la mer par le Canal de Totchè. Au cours de cette période, la concentration à l'IBCG est anormalement très élevée; donc en plus de l'apport des eaux de mer, les déchets de l'IBCG contiennent cet élément.

\section{Le calcium et le magnésium (la dureté de} l'eau) : En dehors de la période de basses eaux, les concentrations de calcium sont supérieures aux concentrations de magnésium. Par contre, lors de la période de basses eaux, les concentrations de calcium sont inférieures à celles de magnésium; ceci est caractéristique des eaux de mer (Gaujous, 1995). En effet, c'est uniquement au cours de cette période que la salinité n'est pas nulle à tous les points sauf à Bouédomey. Ceci confirme l'hypothèse selon laquelle les eaux de mer arrivent à tous les autres points de la lagune et influencent leurs caractéristiques physicochimiques.

Les nutriments et les matières organiques : Les teneurs en phosphore total (PT), en Indice permanganate (IP) et la DCO sont présentées dans le Tableau 4 et la Figure 4.

Le NTK (NTK = Norganique + Nammoniacal) : L'azote ne semble pas poser de problème. Les analyses de NTK n'ont pas été faites lors de la première campagne. Pendant les périodes de pluies et de hautes eaux, les concentrations sont inférieures à 0,5 $\mathrm{mgN} / \mathrm{L}$. Les concentrations obtenues au cours de la période des basses eaux montrent que le Canal de Totchè et l'IBCG sont plus riches en NTK.

Le phosphore total: Toutes les valeurs moyennes annuelles dépassent $100 \mu \mathrm{gP} / \mathrm{L}$.

La lagune est alors hypereutrophe selon l'Organisation de Coopération et Développement Economique (OCDE). Les concentrations élevées sont obtenues au début de la saison des pluies et tout comme pour le NTK, la concentration la plus élevée est obtenue à la SONICOG. Ces résultats montrent que les eaux de ruissellement drainent vers la lagune le phosphore et l'azote. 
La Demande Chimique en Oxygène (DCO) : Les valeurs moyennes annuelles dépassent largement la limite supérieure du bon état écologique défini par la Directive Cadre Européenne pour l'eau.

La valeur la plus élevée est obtenue à Bouédomey lors de la période des basses eaux, ce résultat est identique à celui de l'indice permanganate. On note également des valeurs relativement élevées au Canal de Totchè. Les valeurs obtenues lors de la période des hautes eaux sont toutes inférieures à $40 \mathrm{mgO}_{2} / \mathrm{L}$ qui est la limite de détection de l'appareil.

\section{Diagnostic de l'eutrophisation}

L'outil de diagnostic de l'eutrophisation utilisé dans cette étude a été utilisé dans plusieurs études de lagunes (Ifremer, 2002 et 2004). Il a permis de connaitre l'état de ces lagunes et de les classer.

Le diagnostic établi montre que le complexe lagunaire est dans un mauvais état.

\section{L'analyse des donnees \\ Matrice de corrélations}

Le Tableau 7 montre les coefficients de corrélation entre les différents éléments minéraux. Le coefficient de corrélation détermine la relation entre deux variables et mesure l'intensité de ce lien. Il varie entre -1 et +1 . Lorsqu'il est égal à 1 , il indique un lien parfait entre les variables et lorsqu'il est égal à 0 , il indique une absence de lien. Le signe + signifie que la relation est proportionnelle alors que le signe - signifie que la relation est inversement proportionnelle. Le calcul du coefficient de corrélation permet d'avoir une idée sur les éventuelles relations entre éléments : origine commune, distribution homogène, comportement identique vis-à-vis des processus physicochimiques, fractionnement similaire, influence de l'un sur l'autre.

Le logiciel utilisé est SPSS 12 et les coefficients sont calculés selon la méthode de Bravais-Pearson.

On constate qu'il y a une forte relation entre les sulfates et les nitrates. Leurs présences sont probablement liées à la pollution organique.

On note une forte corrélation entre le $\mathrm{Mg}^{2+}, \mathrm{Ca}^{2+}, \mathrm{K}^{+}$et le $\mathrm{Cl}^{-}$. La relation entre le $\mathrm{Ca}^{2+}$ et le $\mathrm{K}^{+}$est très importante $(\mathrm{r}=1)$. Ces différents éléments sont donc sous forme de chlorures et sont apportés par le lac Nokoué.

On note une relation entre le $\mathrm{Na}^{+}$et le $\mathrm{Cl}^{-}$. On note une corrélation négative entre le $\mathrm{NH}_{4}{ }^{+}$et $\mathrm{l}^{\prime} \mathrm{O}_{2}$. Plus la concentration en $\mathrm{NH}_{4}{ }^{+}$ augmente plus la concentration en oxygène diminue ; le $\mathrm{NH}_{4}{ }^{+}$appauvrit donc le milieu en oxygène lors de sa dégradation.

Une corrélation positive entre la conductivité et le $\mathrm{K}^{+}$, le $\mathrm{Mg}^{2+}$ et le $\mathrm{Ca}^{2+}$. Ces trois éléments majeurs et les chlorures sont les éléments les plus abondants des éléments minéraux de la lagune.

On note une relation entre le $\mathrm{Mg}^{2+}$ et le $\mathrm{Na}^{+}$.

\section{La classification numérique des six sites}

La classification numérique des six sites de prélèvement en fonction de leurs compositions en éléments majeurs permet d'obtenir deux groupes.

Le premier est constitué de Bouédomey, de Beaurivage, de Djassin, de Douane Topka et de l'IBCG.

Le deuxième est constitué uniquement du Canal de Totchè. En effet, le Canal de Totchè, qui est le point de communication entre le lac Nokoué et la lagune de PortoNovo, est caractérisé par des valeurs très élevées en éléments majeurs. 
Tableau 1 : Sites de prélèvements des eaux de la lagune.

\begin{tabular}{|c|c|c|c|}
\hline Sites & Nom & Raisons du choix du site & $\begin{array}{l}\text { Coordonnées } \\
\text { géographiques }\end{array}$ \\
\hline E1 & $\begin{array}{l}\text { L'embarcadère de } \\
\text { Djassin }\end{array}$ & $\begin{array}{l}\text { Il permet d'apprécier l'influence du trafic } \\
\text { humain et des marchandises partant de ce } \\
\text { point à destination des Aguégués. }\end{array}$ & $\begin{array}{l}\text { N06 } 28^{\circ} 28.6^{\prime \prime} \\
\text { E002 }^{\circ} 35^{\prime} 27.7^{\prime}\end{array}$ \\
\hline $\mathbf{E 2}$ & Bouédomey & $\begin{array}{l}\text { Situé au milieu de la lagune ce point } \\
\text { constituait une source } \\
\text { d'approvisionnement en eau des } \\
\text { populations des Aguégués, ses eaux ne } \\
\text { sont jamais salées selon les propos des } \\
\text { riverains. }\end{array}$ & $\begin{array}{l}\text { N06 } 29^{\prime} 49.9^{\prime \prime}, \\
\text { E002'34'13.3", }\end{array}$ \\
\hline $\mathbf{E 3}$ & Canal de Totchè & $\begin{array}{l}\text { C'est par ce point que communique le Lac } \\
\text { Nokoué avec la lagune. Il permettra de } \\
\text { mesurer donc l'apport du Lac Nokoué }\end{array}$ & $\begin{array}{l}\text { N06 } 27^{\prime} 14.5^{\prime \prime} \\
\text { E002 }^{\circ} 34^{\prime} 51.3^{\prime \prime}\end{array}$ \\
\hline E4 & Douane Topka & $\begin{array}{l}\text { Il permet de mesurer l'apport du petit } \\
\text { marché situé non loin du pont de Porto- } \\
\text { Novo. }\end{array}$ & $\begin{array}{l}\text { N06 } 27^{\circ} 59.4^{\prime \prime} \\
\text { E002 } 37 ' 21.4^{\prime \prime}\end{array}$ \\
\hline E5 & IBCG & $\begin{array}{l}\text { IBCG est une savonnerie située non loin } \\
\text { de la lagune. Donc il s'agit de mesurer } \\
\text { l'influence d'éventuels rejets. }\end{array}$ & $\begin{array}{l}\text { N06 } 27^{\circ} 45.1^{\prime \prime}, \\
\text { E002 } 38^{\prime} 25.4^{\prime \prime}\end{array}$ \\
\hline E6 & Hôtel Beaurivage & C'est un complexe hôtelier & $\begin{array}{l}\text { N06 }^{\circ} 28^{\prime} 14.4^{\prime \prime} \\
\text { E002 }^{\circ} 36^{\prime} 18.4^{\prime \prime}\end{array}$ \\
\hline
\end{tabular}

Tableau 2 : Les conditions chromatographiques.

\begin{tabular}{|c|c|c|}
\hline Les différents composants & Anions & Cations \\
\hline Colonne de guarde & $\begin{array}{l}\text { IonPac AG14A Guard } \\
\text { Column }\end{array}$ & $\begin{array}{l}\text { IonPac CG12A Guard } \\
\text { Column }\end{array}$ \\
\hline Colonne de séparation & $\begin{array}{l}\text { IonPac AS14A } \\
\text { Analytical Column }\end{array}$ & $\begin{array}{l}\text { IonPac CS12A Analytical } \\
\text { Column }\end{array}$ \\
\hline Suprresseur & $\begin{array}{l}\text { ASRS-300 Anion } \\
\text { Suppressor, } 4 \text { mm }\end{array}$ & $\begin{array}{l}\text { CSRS-300 Cation } \\
\text { Suppressor, } 4 \mathrm{~mm}\end{array}$ \\
\hline Eluent & $\begin{array}{l}\text { AS14A Eluent } \\
\text { Concentrate, } 250 \mathrm{ml}\end{array}$ & $\begin{array}{l}\text { Methanesulfonic Acid, } \\
500 \mathrm{ml}\end{array}$ \\
\hline Standard & $\begin{array}{l}\text { Standard 7-Anion II } \\
\text { Reagent, } 100 \mathrm{ml}\end{array}$ & $\begin{array}{l}\text { Combined 6-Cation II } \\
\text { Reagent, } 50 \mathrm{ml}\end{array}$ \\
\hline
\end{tabular}


Tableau 3 : Référence - Domaine de mesure - Fiabilité des méthodes utilisées sur la NOVA60.

\begin{tabular}{llll}
\hline Paramètre & Référence méthode & $\begin{array}{l}\text { Domaine de } \\
\text { mesure }\end{array}$ & $\begin{array}{c}\text { Fiabilité de la } \\
\text { méthode }\end{array}$ \\
\hline $\begin{array}{l}\text { Phosphore et } \\
\text { phosphore total }\end{array}$ & Spectroquant Merck & $0,05-5,00 \mathrm{mg} / \mathrm{L} \mathrm{de}$ & $\pm 0,05 \mathrm{mg} / \mathrm{L}$ \\
\hline Azote total & 1.14543 .0001 & $\mathrm{PO}_{4}-\mathrm{P}$ & $\mathrm{PO}_{4}-\mathrm{P}$ \\
\hline & Spectroquant Merck & $0,5-15,0 \mathrm{mg} / \mathrm{L} \mathrm{de} \mathrm{N}$ & $\pm 0,3 \mathrm{mg} / \mathrm{L}$ \\
\hline
\end{tabular}

Tableau 4 : Valeurs moyennes, Moyennes annuelles, Ecart Type, Coefficient de Dispersion et Médiane.

\begin{tabular}{|c|c|c|c|c|c|c|c|c|c|}
\hline & E1 & $\mathbf{E 2}$ & $\mathbf{E 3}$ & E4 & E5 & E6 & Moyenne & Ecart Type & CD (\%) \\
\hline PT (mg/L) & 1,34 & 0,31 & 3,4 & 2,023 & 6,05 & 1,67 & 2,29 & 3,47 & 152,64 \\
\hline IP (mg/L) & 3,83 & 5,66 & 5,25 & 5,89 & 5,79 & 5,31 & 5,29 & 1,92 & 36,36 \\
\hline $\begin{array}{l}\text { DCO } \\
\left(\mathrm{mgO}_{2} / \mathrm{L}\right)\end{array}$ & 101 & 142 & 137 & 109 & 113 & 103 & 117 & 45 & 39 \\
\hline
\end{tabular}

Tableau 5 : Grille de diagnostic pour les sédiments du complexe lagunaire lac Nokoué-lagune de Porto-Novo.

\begin{tabular}{lrrrrrrr|r}
\hline Variable & Très bon & Bon & Moyen & Médiocre & Mauvais \\
\hline MO $(\%)$ & & 3,5 & 4,4 & 5 & 7,5 & 10 & \\
& & 1 & & & & & & \\
NT $(\mathrm{g} / \mathrm{kg})$ & 0,27 & 400 & 500 & 600 & 7 & 700 & 329500 \\
PT $(\mathrm{mg} / \mathrm{kg})$ & & 400 & & & & & & \\
\hline
\end{tabular}

Tableau 6: Grille de lecture de la colonne d'eau du complexe lagunaire lac Nokoué-lagune de Porto-Novo.

\begin{tabular}{|c|c|c|c|c|c|c|c|c|c|}
\hline Variable & Très bon & & Bon & & Moyen & & Médiocre & & Mauvais \\
\hline$\Delta(\%) \mathrm{O}_{2}$ sat & 0 & 20 & & 30 & & 40 & & 50 & 57 \\
\hline TUR (NTU) & 0 & 5 & & 10 & & 25 & 30 & 40 & \\
\hline $\mathrm{PO}_{4}^{2-}(\mu \mathrm{M})$ & 0 & 0,3 & & 1 & & 1,5 & & 4 & 4376 \\
\hline NITRI $(\mu \mathrm{M})$ & 0 & 0,3 & & 0,5 & & 0,8 & & 1 & 368 \\
\hline NITRA $(\mu \mathrm{M})$ & 0 & 1 & & 3 & & 5 & & 10 & 110 \\
\hline $\operatorname{AMMO}(\mu \mathrm{M})$ & 0 & 1,1 & & 3 & & 5 & & 10 & 717 \\
\hline
\end{tabular}




\begin{tabular}{lccccc|l}
\hline Chla $\left(\mathrm{mg} / \mathrm{m}^{3}\right)$ & 0 & 5 & 7 & 10 & 20 & 23 \\
NT $(\mu \mathrm{M})$ & 0 & 50 & 75 & 100 & 120 & 3125 \\
& & 0,8 & 1,5 & 2,5 & 4,5 & 2291 \\
PT $(\mu \mathrm{M})$ & 0 & 0,8 & \\
\hline
\end{tabular}

Tableau 7 : Matrice de corrélations.

\begin{tabular}{|c|c|c|c|c|c|c|c|c|c|c|}
\hline & $\mathrm{Cl}^{-}$ & $\mathrm{NO}_{2}^{-}$ & $\mathrm{NO}_{3}^{-}$ & $\mathrm{PO}_{4}{ }^{2-}$ & $\mathrm{SO}_{4}{ }^{2-}$ & $\mathrm{Na}^{+}$ & $\mathbf{N H}_{4}{ }^{+}$ & $\mathbf{K}^{+}$ & $\mathbf{M g}^{2+}$ & $\mathrm{Ca}^{2+}$ \\
\hline $\mathrm{Cl}^{-}$ & 1 & -.017 & .078 & -.022 & .142 & $.967(* *)$ & -.193 & $.969(* *)$ & $.967(* *)$ & $.971(* *)$ \\
\hline \multirow[t]{2}{*}{$\mathrm{NO}_{2}^{-}$} & & & - & & & & & & & \\
\hline & & 1 & .162 & -.274 & $.512\left(^{*}\right)$ & .161 & -.109 & .140 & .158 & .137 \\
\hline $\mathrm{NO}_{3}{ }^{-}$ & & & 1 & $.478(*)$ & $.526(* *)$ & -.025 & -.355 & -.028 & -.026 & -.047 \\
\hline $\mathrm{PO}_{4}{ }^{2-}$ & & & & 1 & -.131 & -.122 & -.278 & -.114 & -.123 & -.134 \\
\hline $\mathrm{SO}_{4}{ }^{2-}$ & & & & & 1 & .223 & -.174 & .199 & .220 & .177 \\
\hline $\mathbf{N a}^{+}$ & & & & & & 1 & -.208 & .246 & $.528(* *)$ & .266 \\
\hline $\mathrm{NH}_{4}^{+}$ & & & & & & & 1 & -.243 & -.162 & -.313 \\
\hline $\mathbf{K}^{+}$ & & & & & & & & 1 & $.953(* *)$ & $1.000(* *)$ \\
\hline $\mathbf{M g}^{2+}$ & & & & & & & & & 1 & $.960(* *)$ \\
\hline $\mathrm{Ca}^{2+}$ & & & & & & & & & & 1 \\
\hline
\end{tabular}

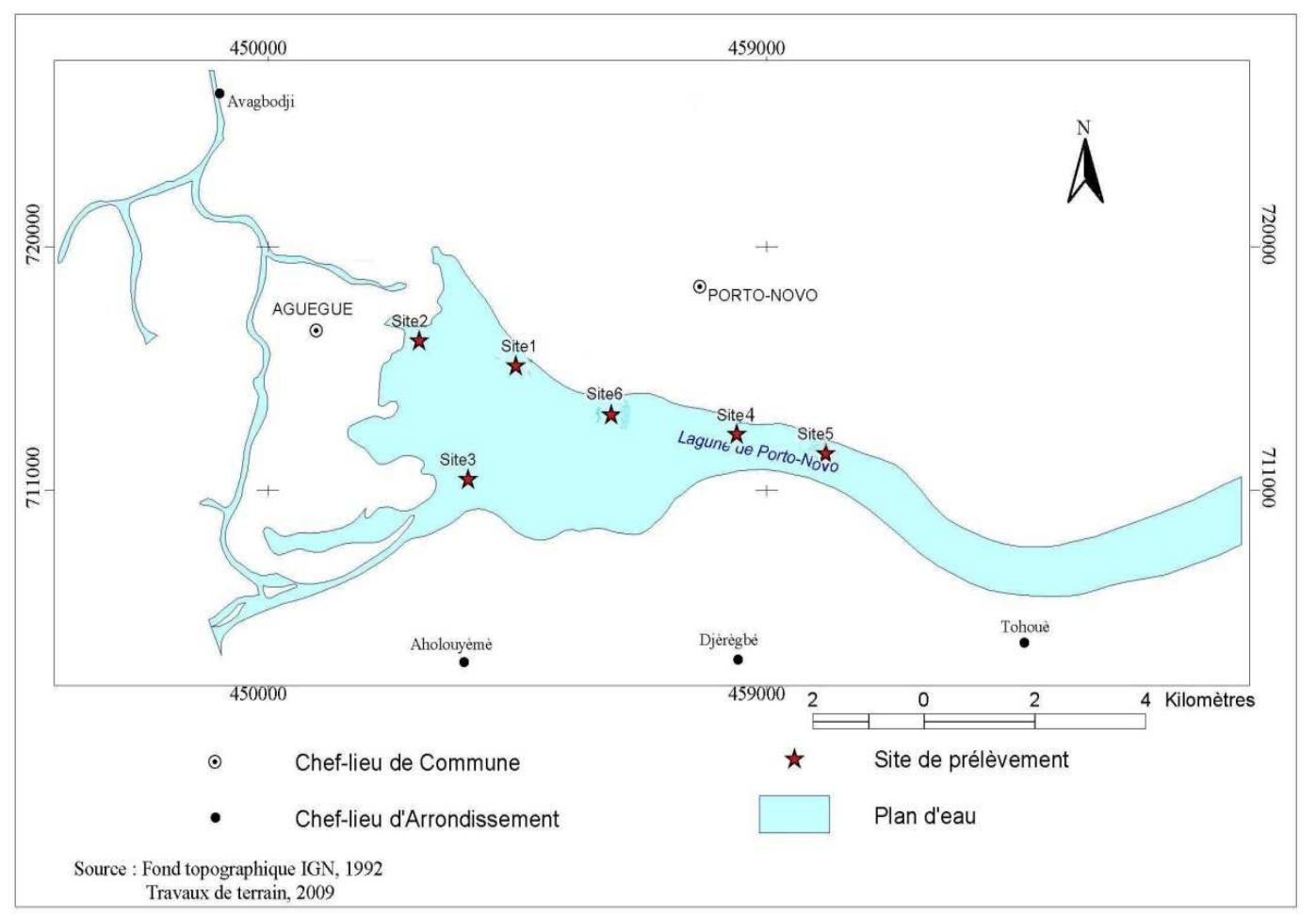

Figure 1: Répartition des sites de prélèvement. 


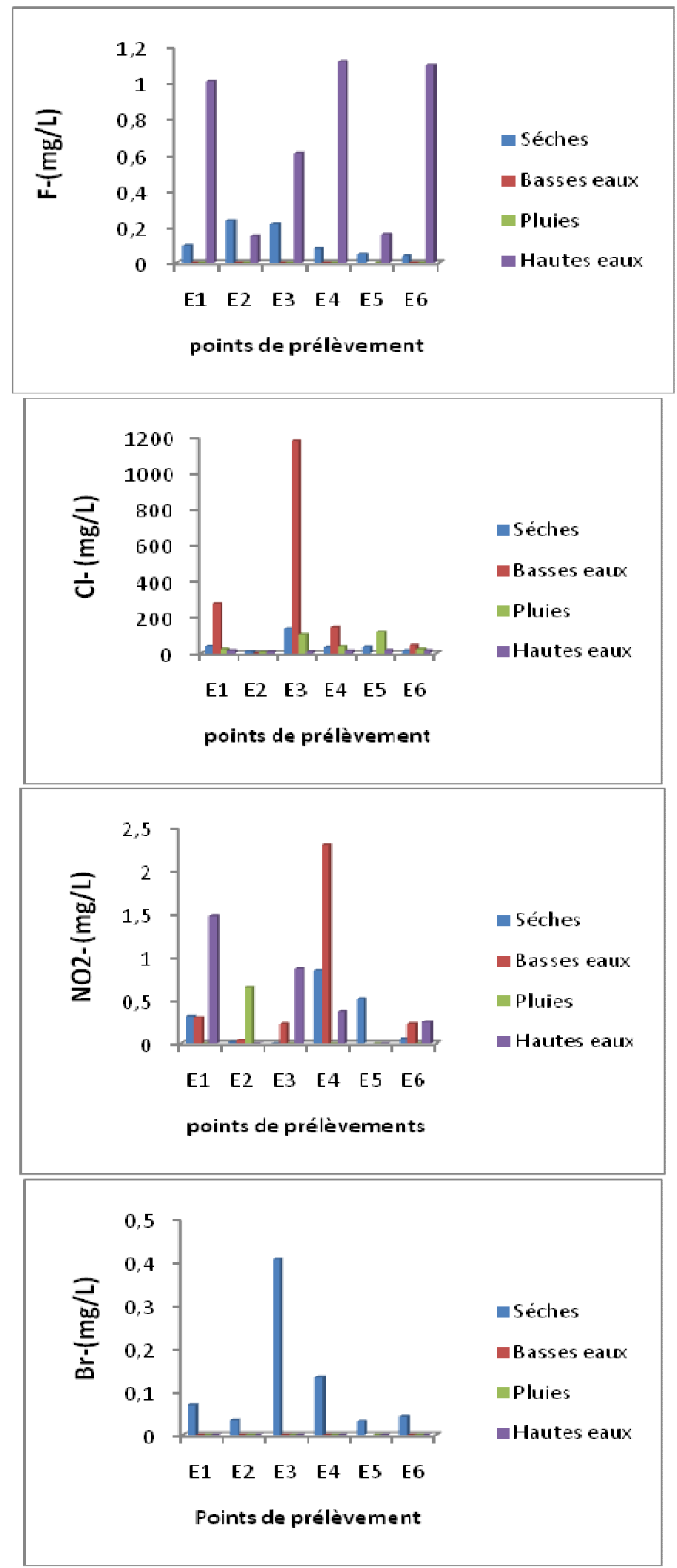




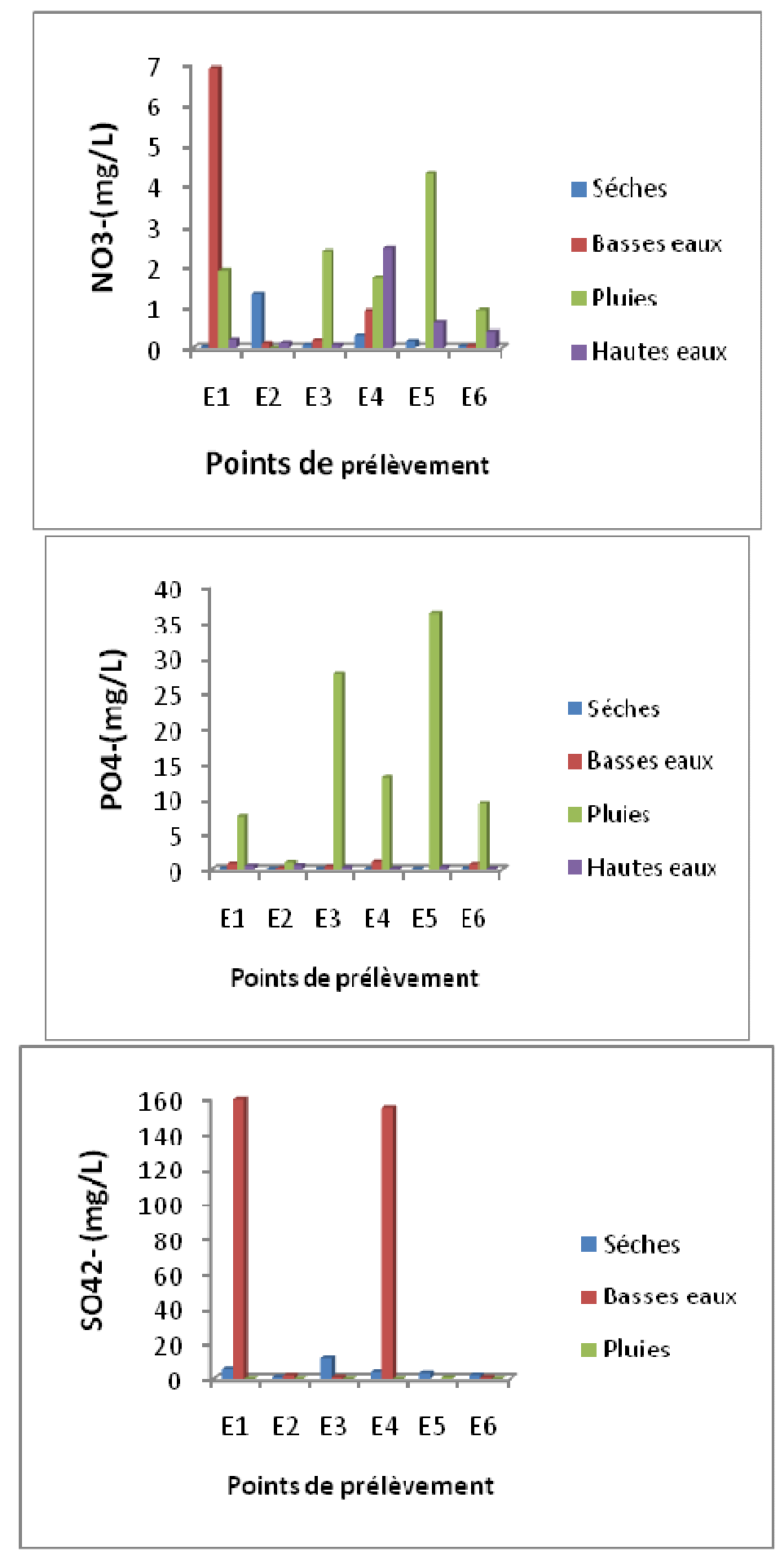

Figure 2: Variations saisonnières des anions de la lagune. 

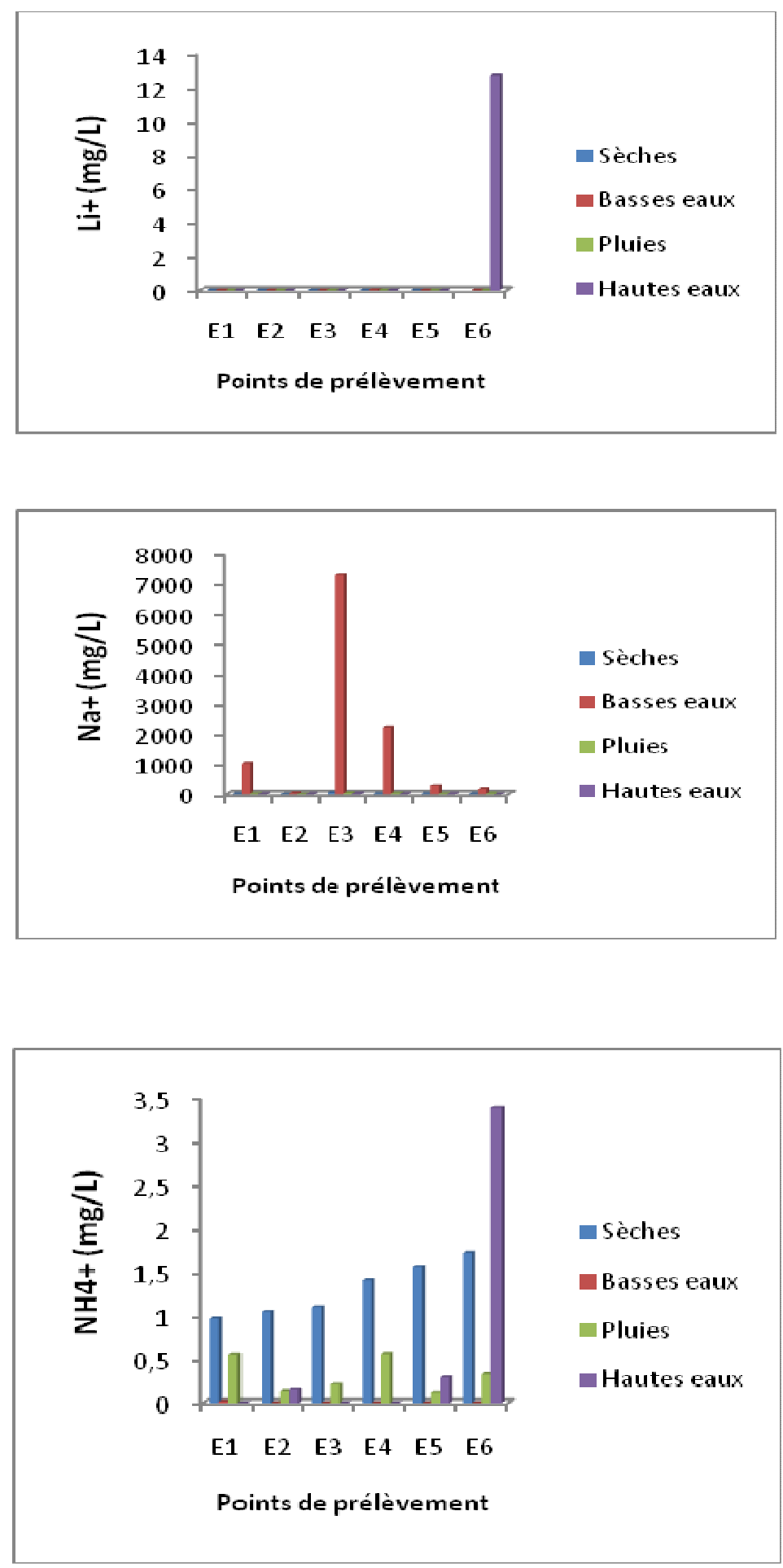

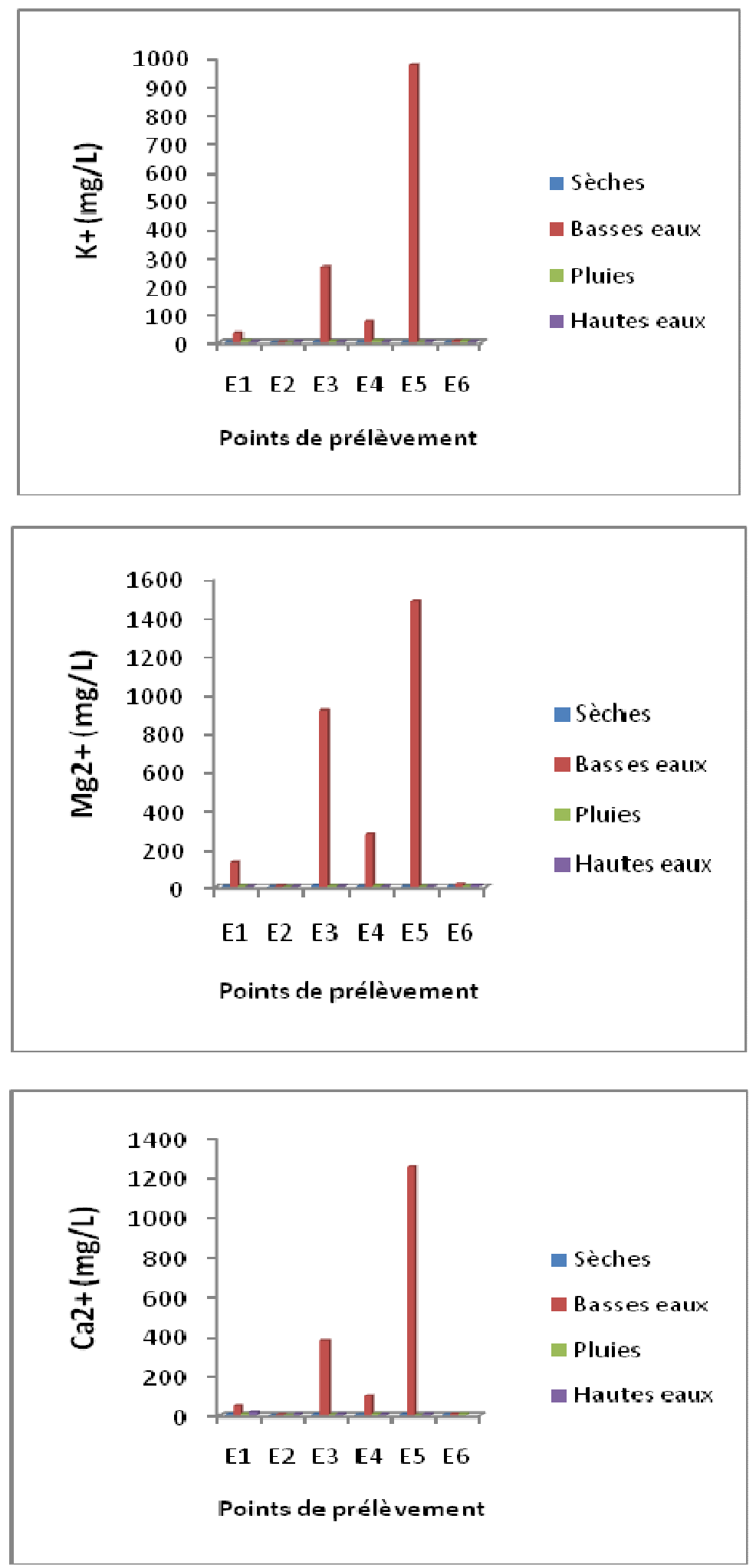

Figure 3: Variations saisonnières des cations de la lagune. 


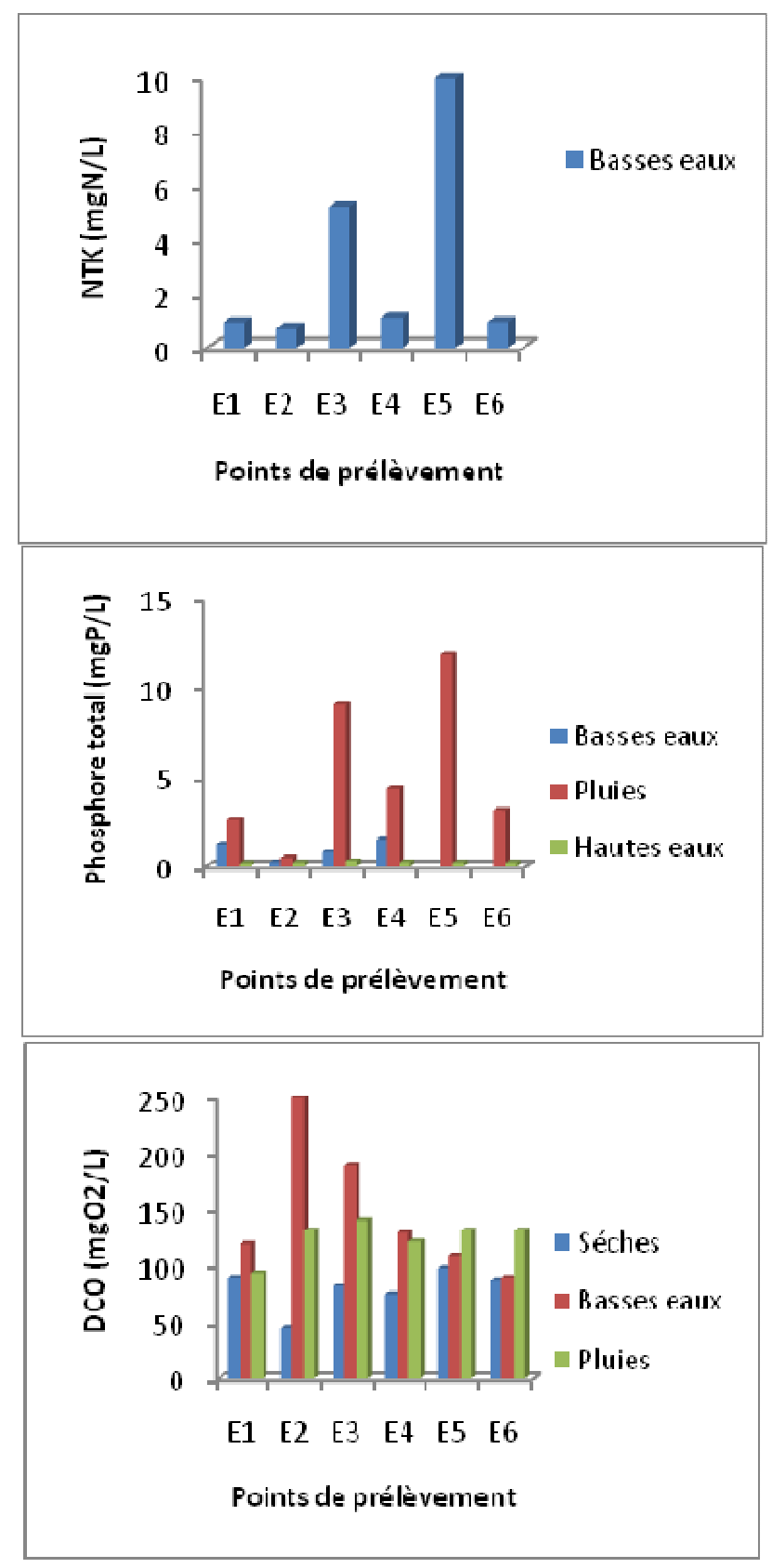

Figure 4 : Variations saisonnières des nutriments et DCO de la lagune.

\section{DISCUSSION}

Le phosphore n'est pas toxique pour les organismes aquatiques mais ses effets secondaires tels que l'eutrophisation et l'épuisement de l'oxygène sont inquiétants.
En effet, l'apport du phosphore dans un milieu aquatique entraine une augmentation de la productivité de la biomasse des végétaux et des algues qui a des effets indésirables tels que : l'accroissement de la turbidité, 
l'augmentation des apports de matière organique qui mène à une sédimentation élevée, la privation d'oxygène (Mason, 1991; Environnement Canada, 2004). Lorsque la croissance excessive de végétaux s'étend à certaines cyanobactéries, il peut y avoir production de toxines, qui exposent la vie aquatique, le bétail et la santé humaine à un risque accru (Chambers et al., 2001). L'envahissement d'un lac par les phytoplanctons ou macrophytes dû à une concentration élevée du phosphore pose un véritable problème esthétique et écologique. La dégradation de ces macrophytes utilise l'oxygène du milieu et conduit donc à l'appauvrissement en oxygène. De même, leur développement excessif dans les milieux eutrophes conduit à une réduction de la transparence qui va alors limiter l'activité photosynthétique (Mama, 2010).

La lagune de Porto-Novo connaît un phénomène d'hyper-eutrophisation. C'est aussi le cas du lac Nokoué (Mama, 2010) qui communique avec la lagune de Porto-Novo. Il arrive parfois que les macrophytes, les nénuphars surtout, bloquent ou rendent difficiles la navigation. Ce phénomène est imputable aux valeurs très élevées de phosphore car les teneurs en NTK sont basses. La teneur moyenne en orthophosphates des eaux de la lagune est supérieure à celle enregistrée (39 $\mu \mathrm{g} / \mathrm{L})$ par Rapin et al. (1999) dans le lac Léman qui connaissait une eutrophisation maximale et dans le lac Ichkeul, où la teneur moyenne de $50 \mu \mathrm{g} / \mathrm{L}$ a été considérée comme un signe d'eutrophisation (M'Barek, 1995). Cette comparaison a permis d'avancer que la lagune peut être classée parmi les milieux hypereutrophes.

Les macrophytes utilisent de grandes quantités d'oxygène et empêchent la lumière de pénétrer dans l'eau. L'eau devient donc invivable pour les autres organismes et surtout pour les poissons. De même, la dégradation chimique de ces algues nécessite de l'oxygène ; ce qui explique les valeurs élevées de DCO. Cette dégradation appauvrit donc la lagune en oxygène comme l'ont montré les faibles teneurs en oxygène dissous (Chouti et al., 2010a).

Aussi, lorsqu'on compare les valeurs moyennes annuelles des nitrates $(1,10 \mathrm{mg} / \mathrm{L})$, des nitrites $(0,37 \mathrm{mg} / \mathrm{L})$ et des ions ammonium $(0,72 \mathrm{mg} / \mathrm{L})$ de la lagune de PortoNovo avec celles de la lagune de Fresco (Yacoub et al., 2008), on constate que la teneur moyenne des nitrates de la lagune est inférieure à celle de la lagune de Fresco $(21,31 \mathrm{mg} / \mathrm{L})$ alors que les teneurs des nitrites et ions ammonium sont supérieures à celles de la lagune de Fresco $\left(0,014 \mathrm{mg} / \mathrm{L}\right.$ de $\mathrm{NO}_{2}{ }^{-}$et $0,06 \mathrm{mg} / \mathrm{L} \mathrm{de} \mathrm{NH}_{4}{ }^{+}$). Ceci confirme le fait que la lagune de Porto-Novo soit déficitaire en oxygène et soit par conséquent un milieu réducteur.

Les sources exogènes de ces apports en nutriments sont les eaux usées domestiques et surtout les eaux de ruissellement comme le témoignent les valeurs élevées observées au début de la saison des pluies. De plus, des résultats antérieurs ont montré que les sédiments de la lagune sont contaminés par le phosphore (Chouti et al., 2010b); ces sédiments constituent donc une source endogène de phosphore.

Les concentrations de chlorures et de sodium sont plus élevées au cours de la période de basses eaux. De même, les concentrations de magnésium sont supérieures à celles de calcium au cours de cette même période, ce qui caractérise les eaux marines (Gaujous, 1995). Donc, pendant cette période la lagune reçoit des eaux salées par l'intermédiaire du lac Nokoué.

\section{Conclusion}

La lagune de Porto-Novo est une lagune hypereutrophe et en mauvais état et les conséquences se font déjà sentir. On observe des macrophytes à divers endroits et la jacinthe d'eau (les nénuphars) rend parfois la navigation impossible.

Les eaux urbaines et surtout les eaux de ruissellement constituent les principales sources d'apport en phosphore de la lagune.

Ces résultats montrent l'urgence de la mise en place d'un système de gestion et de 
contrôle des eaux usées de la ville avant le déversement dans la lagune.

\section{REMERCIEMENTS}

Les auteurs tiennent à remercier tout le personnel de l'Agence des Musulmans d'Afrique (AMA) pour avoir financé cette étude.

\section{RÉFÉRENCES}

Abba E, Nassali H, Benabid M, Ayadi R, Ibaoui H. 2008. Contribution à l'étude physicochimique de l'écosystème lacustre Dayet Aoua au Maroc. Afrique Science, 04(2): 306 - 317.

CCMRE (Conseil canadien des ministres des ressources et de l'environnement). 1987. Recommandations pour la qualité des eaux au Canada. Préparées par le Groupe de travail sur les recommandations pour la qualité des eaux.

Chambers PA, Kent R, Charlton MN, Guy M, Gagnon C, Roberts E, Grove E, Foster N. 2001. Les éléments nutritifs et leurs effets sur l'environnement au Canada. Environnement Canada, p. 271.

Chouti W, Mama D, Alapini F. 2010a. Etude des variations spatio-temporelles de la pollution des eaux de la lagune de PortoNovo (sud Bénin). Int. J. Biol. Chem. Sci., 4(4): 1017-1029.

Chouti W, Mama D, Changotade O, Alapini F, Boukari M. 2010b. Étude des éléments traces métalliques contenus dans les sédiments de la lagune de Porto-Novo (Sud Bénin). J. Applied. Biosci., (34): 2186-2197.

Christel T. 2009. Le risque d'eutrophisation des lagunes Méditerranéennes : Le cas de la lagune de Thau (Hérault). Mémoire de Master 1, Université Paul Valéry Montpellier III, p. 121.

Circulaire DCE $\mathrm{n}^{\circ}$ 2005-12 du 28/07/05) relative à la définition du «bon état » et à la constitution des référentiels pour les eaux douces de surface (cours d'eau, plans d'eau), en application de la directive européenne 2000/60/DCE du 23 octobre 2000, ainsi qu'à la démarche à adopter pendant la phase transitoire (2005-2007).

Conseil canadien des ministres de l'environnement. 2003. Recommandations canadiennes pour la qualité des eaux en vue de protéger la vie aquatique : l'ion nitrate. In Recommandations canadiennes pour la qualité de l'environnement, 1999, Conseil canadien des ministres de l'environnement, Winnipeg.

Environnement Canada. 2004. Canadian guidance framework for the management of phosphorus in freshwater systems. Scientific Supporting Document. Bureau national des recommandations et des normes, Direction générale de la coordination et des politiques relatives à l'eau, Environnement Canada, Ottawa (Ontario).

Gaujous D. 1995. La Pollution des Milieux Aquatiques. Aide-mémoire ( $2^{\text {ème } e ́ d n, ~}$ revue et augmentée). Technique et Documentation - Lavoisier.

Ifremer. 2002. Direction de l'Environnement et de l'Aménagement Littoral, Laboratoire de Sète, Rapport du Réseau Lagunaire du Languedoc-Roussillon, Bilan des résultats 2001.

Ifremer. 2004. Direction de l'Environnement et de l'Aménagement Littoral, Laboratoire Environnement- Ressources $\mathrm{du}$ Languedoc-Roussillon, Rapport du Réseau Lagunaire du LanguedocRoussillon, Bilan des résultats 2003.

Kouassi AM. 2005. Hydrochimie et qualité des eaux de deux lagunes tropicales (Ebrié et Grand Lahou). Thèse de doctorat, Université de Cocody, p.147.

M'Barek N. 1995. Impact des ouvrages sur l'équilibre de l'écosystème : lac Ichkeul. Rapport de DEA, Université de Tunis II, p. 100.

Mama D. 2010. Méthodologie et résultats du diagnostic de l'eutrophisation du lac Nokoué (Benin). Thèse de doctorat, Université de Limoges, p. 157.

Mason C. 1991. Biology of Freshwater Pollution $\left(2^{\text {nd }}\right.$ edn). Longman Scientific 
and Technical, Longman Group UK Limited: p. 351.

Mohamedou E, Lebkiri A, Rifi H, Lebkiri M, Fadli M, Pontie M, Mahmoud A, Fagel L. 2008. Typologie physico-chimique et métallique des eaux du fleuve Sénégal au niveau de la ville de Rosso (Mauritanie). Afrique Science, 04(3): 394 - 409.

OCDE (OECD). 1982. Eutrophisation des Eaux. Méthodes de Surveillance, d'Evaluation et de Lutte. Organisation de Coopération et de Développement Economiques : Paris ; 164.

Rapin A, Blanc P, Corvec C. 1989. Influence des apports sur le stock de phosphore dans le lac Léman et sur l'eutrophisation. Revue Sciences de l'Eau, (2): 721-737.
Ruiz F, Abad M, Galan E, Gonzalez I, Aguila I, Olias M, Gomez Ariza J, Cantano M. 2006. The present environmental scenarion of El Melah Lagoon (NE Tunisia and its evolution to a future sabkha. Journal of African Earth Sciences, (44): 289-302.

Truelson R. 1997. Water quality criteria for dissolved oxygen. Préparé pour le British Columbia's Ministry of Environment, Lands and Parks, Water Management Branch, Victoria.

Yacoub I, Aka K, Dongui B, Biemi J. 2008. Caractéristiques physico-chimiques d'une lagune côtière tropicale : lagune de Fresco (Côte d'Ivoire). Afrique Science 04(3): 368-393. 